\title{
Transmission of SARS-CoV-2 by children: a rapid review, 30 December 2019 to 10 August 2020
}

Barbara Clyne $^{1,2}$, Karen Jordan ${ }^{1}$, Susan Ahern ${ }^{1}$, Kieran A Walsh ${ }^{1}$, Paula Byrne ${ }^{1}$, Paul G Carty ${ }^{1}$, Linda Drummond ${ }^{1}$, Kirsty K

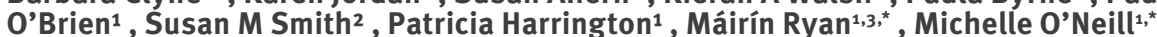

1. Health Information and Quality Authority, Dublin, Ireland

2. Department of General Practice, RCSI University of Medicine and Health Sciences, Dublin, Ireland

3. Department of Pharmacology \& Therapeutics, Trinity College Dublin, Trinity Health Sciences, St James's Hospital, Dublin, Ireland

* These authors contributed equally and share last authorship

Correspondence: Barbara Clyne (barbaraclyne@rcsi.com)

Citation style for this article:

Clyne Barbara, Jordan Karen, Ahern Susan, Walsh Kieran A, Byrne Paula, Carty Paul G, Drummond Linda, O’Brien Kirsty K, Smith Susan M, Harrington Patricia, Ryan Máirín, O’Neill Michelle. Transmission of SARS-CoV-2 by children: a rapid review, 30 December 2019 to 10 August 2020. Euro Surveill. $2022 ; 27$ (5):pii=2001651.

https://doi.org/10.2807/1560-7917.ES.2022.27.5.2001651

Background: The role of children in the transmission of SARS-CoV-2 during the early pandemic was unclear. Aim: We aimed to review studies on the transmission of SARS-CoV-2 by children during the early pandemic. Methods: We searched MEDLINE, Embase, the Cochrane Library, Europe PubMed Central and the preprint servers medRxiv and bioRxiv from 30 December 2019 to 10 August 2020. We assessed the quality of included studies using a series of questions adapted from related tools. We provide a narrative synthesis of the results. Results: We identified 28 studies from 17 countries. Ten of 19 studies on household and close contact transmission reported low rates of child-to-adult or child-to-child transmission. Six studies investigated transmission of SARS-CoV-2 in educational settings, with three studies reporting 183 cases from 14,003 close contacts who may have contracted COVID-19 from children index cases at their schools. Three mathematical modelling studies estimated that children were less likely to infect others than adults. All studies were of low to moderate quality. Conclusions: During the early pandemic, it appeared that children were not substantially contributing to household transmission of SARS-CoV-2. School-based studies indicated that transmission rates in this setting were low. Large-scale studies of transmission chains using data collected from contact tracing and serological studies detecting past evidence of infection would be needed to verify our findings.

\section{Introduction}

The coronavirus disease (COVID-19) pandemic is a global public health emergency, with over five million cases and over 1.5 million deaths worldwide at time of writing (December 2020) [1,2]. During the early pandemic, in the absence of effective treatments or vaccinations, efforts to reduce transmission were the most effective means of tackling the pandemic. Understanding the burden and transmission of severe acute respiratory syndrome coronavirus 2 (SARS-CoV-2) in children is essential for implementing appropriate public health measures and taking decisions about the provision of childcare and education. Adults and children of any age can be infected with SARS-CoV-2, however, paediatric SARS-CoV-2 infection is often milder, most children with symptomatic COVID-19 have a low risk of death or hospitalisation, and a large proportion are likely to be asymptomatic [3-5]. Initially, reported cases of COVID-19 in paediatric populations accounted for a small percentage of all diagnosed cases. International data suggested that children and young people accounted for $4 \%$ to $7 \%$ of reported cases $[6,7]$. Over time, the proportion of paediatric COVID-19 cases has risen as overall transmission has increased [8]. Fewer cases may have been initially diagnosed in children, leading to potential under-reporting because of initial test capacity constraints. There was an international trend towards restricting or prioritising real-time reverse transcription polymerase chain reaction testing to designated groups such as healthcare workers and or those with a greater risk of complications because of underlying disease. Potential under-reporting could have also been because of a reduction in typical agemixing such as with school contacts $[9,10]$.

There is growing evidence that children are less susceptible to severe SARS-CoV-2 infection than adults [11]. This may relate to differences in the way children interact with their environment and their dynamic developmental physiology and immunity [11]. Lower susceptibility appeared to be confined to those younger than 10-14 years old, while the risk in teenagers appeared to be closer to young adults [11]. A mathematical modelling study using data from six countries 
estimated that susceptibility to infection in individuals under 20 years of age was approximately half that of adults aged over 20 years [12]. The authors argued that low case rates in children and young people could also be explained by age-specific severity i.e. children are more likely to be asymptomatic and experience milder symptoms [12]. This may also represent under-testing and under-reporting in this age group in the early pandemic, as well as issues of potential lower diagnostic accuracy in asymptomatic cases. Many of the early pandemic studies were of low quality and data was limited [11].

Age-related transmissibility of SARS-CoV-2 has become an important public health concern, however, the role that children played in transmission during the early pandemic was unclear.

Our aim was to synthesise the available evidence on how children were contributing to the spread of SARSCoV-2 during the early pandemic.

\section{Methods}

Our team at the Health Information and Quality Authority (HIQA) of Ireland has conducted a series of rapid reviews for a broad range of public health topics related to COVID-19. These followed a standardised protocol developed a priori [13], in line with Cochrane rapid review methodology guidance [14]. The need for rapid reviews arose directly from questions posed by policymakers and expert clinicians supporting the National Public Health Emergency Team. The findings of these reviews have informed the national response to the COVID-19 pandemic in Ireland [15] and have implications for international health policy as well as clinical and public health guidance. Sections of this manuscript have been published on HIQA's website as a white paper [16].

\section{Search strategy}

Searches were conducted in MEDLINE, Embase, Cochrane Library, Europe PubMed Central and two preprint servers medRxiv and bioRxiv. The search terms and detailed search strategy are available in the protocol [13]. Reference lists of included articles were also searched. No language restrictions were applied. The search period was from 30 December 2019 to 10 August 2020.

\section{Inclusion and exclusion criteria}

All potentially eligible papers identified through the search, including preprints, were exported to Endnote X8.2 (Thomson Reuters, New York, United States (US)) and screened for relevance by the review team (BC, KJ, SA, KAW, PB, PGC, LD, KKOB). Any study (regardless of design) that addressed the research question was included as per the inclusion and exclusion criteria presented in Table 1.
Data extraction and quality assessment

For each included study, the study design, participant demographics and clinically relevant data including the numbers of children, index cases, infected contacts and contacts followed up were extracted.

The majority of studies included were case series and modelling studies. No universally accepted quality appraisal tool existed at the time of conducting this review for such study designs. Therefore, a series of questions adapted from related tools were used, as described in the protocol and the Supplement [13]. In view of study heterogeneity a meta-analysis was not possible and findings were synthesised narratively.

\section{Ethical statement}

Ethical approval was not required as this was a review of published literature.

\section{Results}

Characteristics of included studies

A total of 28 studies were included, comprising 19 studies on household and close contact transmission [17-35], six on transmission of SARS-CoV-2 in educational settings [36-41] (Table 2) and three modelling studies estimating age-specific transmissibility of SARS-CoV-2 [42-44] (Table 3). Six studies were from China $[17,18,22,23,25,42]$, three each from the US $[27,29,32]$ and Israel $[33,39,43]$, two from South Korea [28,40], and one report each was from Ireland [37], Switzerland [24], Australia [38], France [19], Italy [20], Vietnam [21], Singapore [45], the Netherlands [34], Thailand [26], Morocco [30], Finland [36], India [31] and New Zealand [44]. A secondary data analysis study included data from China, Singapore, South Korea, Japan and Iran [35]. Seven studies were preprints and had not undergone formal peer review at the time of writing $[25,35,36,40,42-44]$. Sample sizes for included COVID-19 cases in children ranged from 1 to 42,618 , and where reported, contact numbers ranged from 1 to 57,415 in household and close contact transmission studies [19,24,26-29,31,33] and from 119 to over 10,000 in educational settings [36-41]. The age of participants (where reported) ranged from 32 days [20] to younger than 18 years [25]. From the 15 studies that reported demographic information, there was a total of 126 male and 104 female children [17-24,26,28-30,32,35,38].

\section{Study quality}

The 19 primary studies reporting on household and close contact transmission were generally of poor quality in terms of design, as there was a lack of detail on how cases were selected, what the criteria for testing contacts was, what testing was undertaken and how consistently testing was conducted across all contacts (see Figure and Supplement) $[17-25,33,37,38]$.

Three studies had small sample sizes (less than 10) $[17,19,26]$ and seven studies were case reports [2023,28-30]. Two studies used existing public data 
Population, outcomes and study types (POS) framework for inclusion of studies on transmission of SARS-CoV-2 by children, 30 December 2019-10 August 2020

\begin{tabular}{|c|c|c|}
\hline Population & \multicolumn{2}{|c|}{$\begin{array}{l}\text { Children (under the age of } 18 \text { years) with a laboratory-confirmed positive test for SARS-CoV-2; Subgroups of interest: } \\
\text { asymptomatic vs symptomatic (mild, moderate, severe) }{ }^{\mathrm{a}}\end{array}$} \\
\hline Outcome & \multicolumn{2}{|c|}{$\begin{array}{l}\text { Confirmed transmission of SARS-CoV-2 rates } \\
\text { Proportion of which are household transmissions } \\
\text { Mean time to transmission/symptoms onset }\end{array}$} \\
\hline Types of studies & $\begin{array}{l}\text { Include: } \\
\text { Any study that reports on transmission of SARS- } \\
\text { CoV-2 by children }\end{array}$ & $\begin{array}{c}\text { Exclude: } \\
\text { Studies where SARS-CoV-2 was not confirmed with a laboratory } \\
\text { test }\end{array}$ \\
\hline
\end{tabular}

COVID-19: coronavirus disease; SARS-CoV-2: severe acute respiratory syndrome coronavirus 2.

a The disease severity classification by the World Health Organisation at time of writing: https://apps.who.int/iris/handle/10665/330893.

$[25,35]$, i.e. there is potential for double counting of cases across studies. In household studies, transmission was often assessed by self-reporting as to which family member developed symptoms first [24,32,33]. The number of contacts tested, the length of followup, which tests were performed and how long after the index case became positive, test sensitivity and specificity and confounders such as use of face masks and physical distancing were not consistently reported across studies. While household studies provide a unique opportunity to study transmission in a clearly identified cohort of close contacts, it is still possible that transmission events might be missed and errors in ascertaining the direction of transmission may be made. While all six studies in preschool and school settings had small numbers of cases who were children, they followed up a large number of contacts [36-41]. In three of these studies, only symptomatic contacts were followed and tested $[37,38,41]$. Given potential transmission from asymptomatic or mildly symptomatic contacts $[3,4]$, the potential for missing cases cannot be ruled out. In all of these studies, household or community transmission cannot be ruled out for positive cases detected [38-40].

All three modelling studies used household data collected when countries were in various forms of lockdown and there were few child index cases [42-44]. The modelling study by Zhao et al. [42] had a small sample to check the fit of a model and the sample from the seafood market may not be representative of transmission patterns more generally. Data for the model by Dattner et al. [43] did not include dates of infection or information about who the index patient was. The study only used aggregate numbers of infected individuals in two age groups, 0-19-year-olds and 20-yearolds and older) and the majority of the data used were from households with two members. In James et al. [44] data on missing cases and missing transmission routes were estimated using Monte Carlo methods.

\section{Household and close contact transmission} Nineteen studies that examined household and close contact transmission comprised three analyses of surveillance data $[27,31,34]$, eight case series [17$19,24,26,32,33,35]$, seven case reports [20-23,28-30] and one analysis of local heath commissions' public disclosures in China [25].

\section{Children under 2 years of age}

Four case reports which specifically included children under 2 years of age reported no transmission of SARSCoV-2 $[20,21,23,30]$. In three case reports, no transmission from SARS-CoV-2-positive children aged 32 days to 2 years who were being cared for by their mothers was reported $[21,23,30]$. Use of precautions such as face masks was described in two of these studies $[21,30]$. In one case report, no transmission from a SARS-CoV-2-positive infant (aged 32 days) to medical staff was documented [20]. Details on personal protective equipment (PPE) and other precautions used by medical staff were not reported [20].

\section{Children aged $0-10$ years}

Six studies included children aged $0-10$ years $[17,19,22,26,28,35]$, four of which reported transmission of SARS-CoV-2 from this age group. In a case series of 10 patients admitted to children's hospitals in China, Cai et al. [17] reported transmission of SARSCoV-2 from an infant aged 3 months to both parents, who developed symptomatic COVID-19 7 days after taking care of the infant. The authors of the study performing secondary analysis reported 31 SARS-CoV-2 household transmission clusters, of which three were identified as having a paediatric index case including the infant case described by Cai et al. [17] and two children aged 9 and 10 years [35]. In an analysis of these data, assuming a worst case scenario where asymptomatic children were the index case, six of the 28 family clusters would have a paediatric index case [35]. 
TABLE 2A

Characteristics of studies on transmission of SARS-CoV-2 by children, 30 December 2019-10 August 2020 ( $\mathrm{n}=25)$

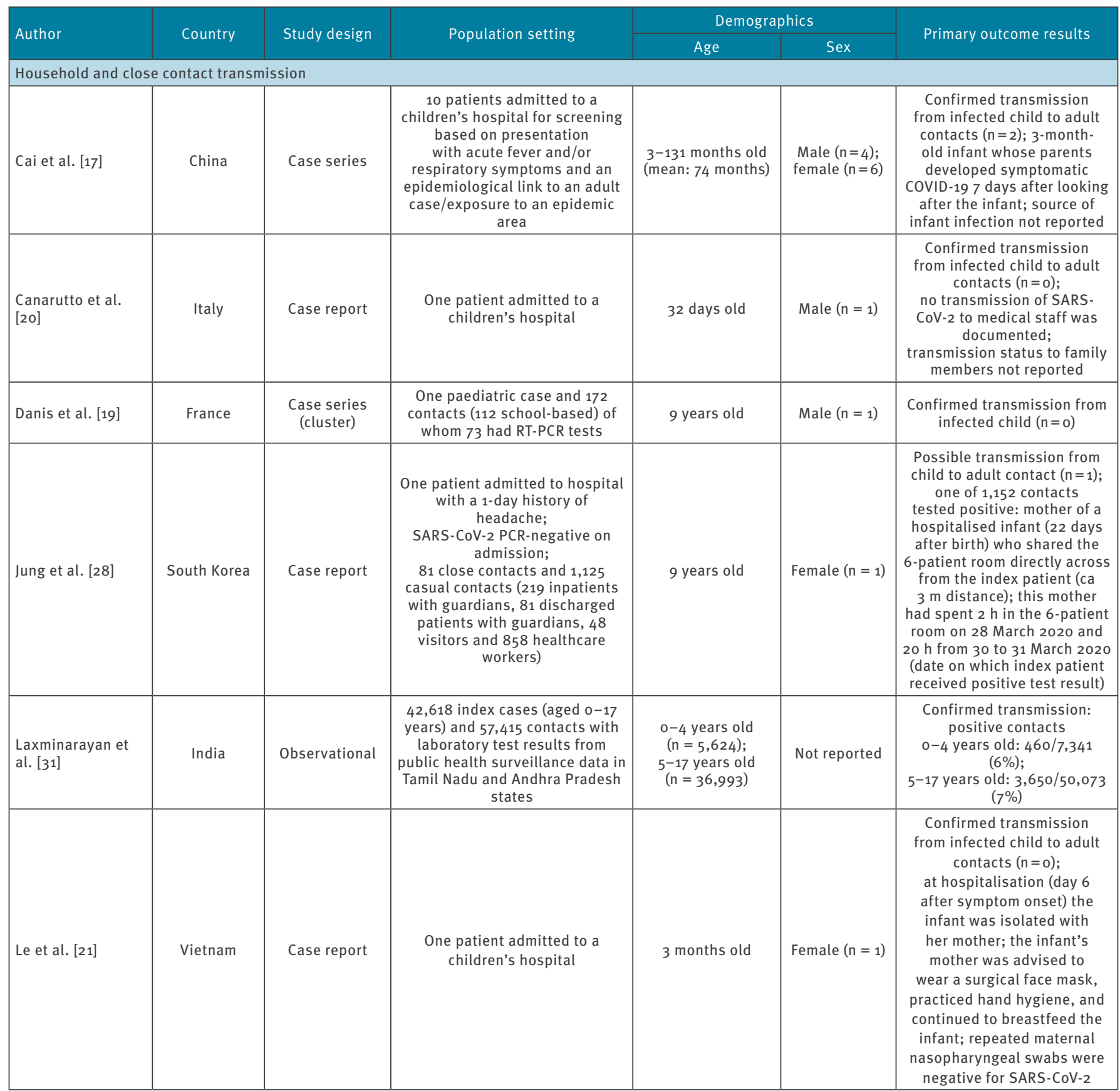

CI: confidence interval; COVID-19: coronavirus disease; ECEC: early childhood education and care; HCW: healthcare workers; HR: hazard ratio; IQR: interquartile range; PPE: personal protective equipment; SARS-CoV-2: severe acute respiratory syndrome coronavirus 2; US: United States.

${ }^{\text {a }}$ One case included here is also included in the case series by Cai et al. [17].

${ }^{\mathrm{b}}$ Article was translated using Google Translate. 
TABLE 2B

Characteristics of studies on transmission of SARS-CoV-2 by children, 30 December 2019-10 August 2020 ( $\mathrm{n}=25)$

\begin{tabular}{|c|c|c|c|c|c|c|}
\hline \multirow{2}{*}{ Author } & \multirow{2}{*}{ Country } & \multirow{2}{*}{ Study design } & \multirow{2}{*}{ Population setting } & \multicolumn{2}{|l|}{ Demographics } & \multirow{2}{*}{ Primary outcome results } \\
\hline & & & & Age & Sex & \\
\hline Lin et al. [22] & China & Case report & $\begin{array}{c}\text { One patient admitted to a } \\
\text { quarantine ward in a local } \\
\text { country hospital; RNA-positive } \\
\text { throat swabs }\end{array}$ & 7 years old & Female $(n=1)$ & $\begin{array}{l}\text { Possible transmission } \\
\text { from infected child to adult } \\
\text { contacts: }(n=1) \text {; } \\
\text { on } 21 \text { January } 2020 \text { the girl's } \\
\text { father drove and then took } \\
\text { a bus to Xiangyang, Hubei } \\
\text { province where he stayed } \\
\text { overnight but did not have } \\
\text { close contact with anybody } \\
\text { except family members. On } 22 \\
\text { January } 2020 \text {, the father drove } \\
\text { himself from Xiangyang, } \\
\text { Hubei to Chongqing city with } \\
\text { the girl, her grandparents, } \\
\text { mother, and } 2 \text { } \square \text { yearDold } \\
\text { brother, arriving in the early } \\
\text { morning of } 23 \text { January } 2020\end{array}$ \\
\hline Lucar et al. [29] & US & Case report & $\begin{array}{c}\text { One index case who was } \\
\text { airlifted to hospital following } \\
\text { a car accident and placed } \\
\text { under general anaesthesia for } \\
\text { orthopaedic surgical intervention } \\
\text { tested SARS-Cov-2-positive on } \\
\text { hospital day 2; } \\
11 \text { HCW present in the operating } \\
\text { room }\end{array}$ & 17 years old & Male $(n=1)$ & $\begin{array}{l}\text { Confirmed transmission from } \\
\text { child to HCW contacts }(n=0) ; \\
\text { 1/11 (assisted with intubation) } \\
\text { developed a dry cough a few } \\
\text { hours post-procedure but } \\
\text { tested SARS-CoV-2 negative; } \\
\text { 10/11 no symptoms } \\
\text { throughout the 14-day } \\
\text { monitoring period and were } \\
\text { not offered SARS-CoV-2 } \\
\text { testing; } \\
\text { HCW wore COVID-19 personal } \\
\text { PPE consisting of N95 mask, } \\
\text { face shield, gowns, and } \\
\text { gloves }\end{array}$ \\
\hline $\begin{array}{l}\text { Mannheim et al. } \\
{[32]}\end{array}$ & US & Case series & $\begin{array}{c}64 \text { paediatric (aged } \leq 17 \text { years) } \\
\text { laboratory-confirmed SARS- } \\
\text { CoV-2 cases reported to the } \\
\text { Chicago Department of Public } \\
\text { Health; } \\
15 \text { households with transmission } \\
\text { data }\end{array}$ & $\begin{array}{l}\text { Median age: } 11 \\
\text { (IQR: 7-16) }\end{array}$ & $\begin{array}{l}\text { Male }(n=36) ; \\
\text { female }(n=28)\end{array}$ & $\begin{array}{l}\text { Confirmed transmission ( } 15 \\
\text { households); } \\
\text { households with child index } \\
\text { case: } 4 / 15 ; \\
\text { child-to-child: } 2 / 15 ; \\
\text { child-to-adult: } 2 / 15 ; \\
\text { number of secondary cases } \\
\text { not reported }\end{array}$ \\
\hline Nassih et al. [30] & Morocco & Case report & $\begin{array}{l}\text { One laboratory-confirmed SARS- } \\
\qquad \text { CoV-2 case }\end{array}$ & 2 years old & Female $(n=1)$ & $\begin{array}{l}\text { Confirmed transmission from } \\
\text { infected child }(\mathrm{n}=0) \text {; } \\
\text { child cared for by mother } \\
\text { for duration of illness ( } 1 \\
\text { month) using airborne and } \\
\text { contact precautions and } \\
\text { mother remained PCR- and } \\
\text { serology-negative }\end{array}$ \\
\hline
\end{tabular}

$\mathrm{CI}$ : confidence interval; COVID-19: coronavirus disease; ECEC: early childhood education and care; HCW: healthcare workers; HR: hazard ratio; IQR: interquartile range; PPE: personal protective equipment; SARS-CoV-2: severe acute respiratory syndrome coronavirus 2; US: United States.

a One case included here is also included in the case series by Cai et al. [17].

${ }^{b}$ Article was translated using Google Translate. 
TABLE 2C

Characteristics of studies on transmission of SARS-CoV-2 by children, 30 December 2019-10 August 2020 ( $\mathrm{n}=25$ )

\begin{tabular}{|c|c|c|c|c|c|c|}
\hline \multirow{2}{*}{ Author } & \multirow{2}{*}{ Country } & \multirow{2}{*}{ Study design } & \multirow{2}{*}{ Population setting } & \multicolumn{2}{|l|}{ Demographics } & \multirow{2}{*}{ Primary outcome results } \\
\hline & & & & Age & Sex & \\
\hline $\begin{array}{l}\text { Posfay-Barbe et } \\
\text { al. [24] }\end{array}$ & Switzerland & Case series & $\begin{array}{l}39 \text { patients aged }<16 \text { years with } \\
\text { SARS-CoV-2 infection (seven } \\
\text { inpatients, } 32 \text { outpatients) and } \\
111 \text { household contacts }\end{array}$ & $\begin{array}{l}\text { Median age } 11.1 \\
(\text { IQR: } 5 \cdot 7-14 \cdot 5)\end{array}$ & $\begin{array}{l}\text { Male }(n=17) \\
\text { female }(n=22)\end{array}$ & $\begin{array}{l}\text { Possible transmission: cluster } \\
\text { with a child developing } \\
\text { symptoms before any other } \\
\text { household contacts: } 3 / 39 ; \\
\text { number of secondary cases: } \\
\text { four (three mothers and one } \\
\text { father) }\end{array}$ \\
\hline Qiu et al. [23] & China & Case report & $\begin{array}{c}\text { One patient admitted to a } \\
\text { children's hospital; RNA-positive } \\
\text { nasopharyngeal and rectal } \\
\text { swabs }\end{array}$ & 8 months old & Male $(n=1)$ & $\begin{array}{l}\text { Confirmed transmission } \\
\text { from infected child to adult } \\
\text { contacts }(n=0)\end{array}$ \\
\hline Somekh et al. [33] & Israel & Case series & $\begin{array}{l}13 \text { family clusters ( } 36 \text { adults, } 58 \\
\text { children); } \\
\text { index case was the first case of } \\
\text { the infection in the family }\end{array}$ & $\begin{array}{l}6 \text { months }-17 \text { years } \\
\text { old }\end{array}$ & Not reported & $\begin{array}{c}\text { Confirmed transmission: } \\
\text { cluster with child index case: } \\
1 / 13 \\
(14 \cdot 5 \text {-year-old male); } \\
\text { transmission from infected } \\
\text { child to adult contacts not } \\
\text { reported; } \\
\text { transmission from infected } \\
\text { child to child contacts not } \\
\text { reported; } \\
\text { number of secondary cases } \\
\text { not reported }\end{array}$ \\
\hline $\begin{array}{l}\text { van der Hoek et } \\
\text { al. }[34]^{\mathrm{a}}\end{array}$ & $\begin{array}{l}\text { The } \\
\text { Netherlands }\end{array}$ & $\begin{array}{l}\text { Prospective } \\
\text { observational } \\
\text { study } \\
\text { (surveillance } \\
\text { data) }\end{array}$ & $\begin{array}{l}732 \text { transmission pairs from } \\
\text { Dutch National Institute } \\
\text { for Public Health and the } \\
\text { Environment national } \\
\text { surveillance data }\end{array}$ & $\begin{array}{l}4-12 \text { years old } \\
\quad(n=9) ; \\
20 \text { years old and } \\
\text { older }(n=31)\end{array}$ & Not reported & $\begin{array}{l}\text { Confirmed transmission from } \\
\text { infected child to child }(n=2) ; \\
\text { the source in one of these } \\
\text { cases was aged } 15-20 \text {-years } \\
\text { old }\end{array}$ \\
\hline $\begin{array}{l}\text { Wongsawat et al. } \\
\text { [26] }\end{array}$ & Thailand & Case series & $\begin{array}{l}\text { Three paediatric COVID } 19 \text { cases } \\
\text { and three adult caregivers }\end{array}$ & 4-8-year-olds & $\begin{array}{l}\text { Male }(n=2) \\
\text { female }(n=1)\end{array}$ & $\begin{array}{c}\text { Confirmed transmission } \\
\text { from infected child to adult } \\
\text { contacts }(\mathrm{n}=0) \text {; } \\
\text { one case was isolated with } \\
\text { his grandfather who was also } \\
\text { infected with COVID-19, and } \\
\text { two cases were isolated with } \\
\text { healthy caregivers; } \\
\text { during isolation, children and } \\
\text { caregivers were advised to } \\
\text { wash hands frequently, not } \\
\text { share personal items and } \\
\text { were provided surgical masks }\end{array}$ \\
\hline Wu et al. [18] & China & Case series & $\begin{array}{c}74 \text { paediatric SARS-CoV-2 cases } \\
\text { admitted in a women's and } \\
\text { children's hospital }\end{array}$ & $\begin{array}{l}\leq 3 \text { months old } \\
(n=7) ; \\
3-6 \text { months old } \\
\quad(n=4) ; \\
6 \text { months }-1 \text { year } \\
\text { old }(n=5) ; \\
1-3 \text { years old } \\
(n=12) ; \\
\text { 3-10 years old } \\
(n=31) ; \\
\text { over } 10 \text { years old } \\
(n=15)\end{array}$ & $\begin{array}{l}\text { Male }(n=44) \\
\text { female }(n=30)\end{array}$ & $\begin{array}{l}\text { Confirmed transmission from } \\
\text { infected child to contacts } \\
\qquad(n=0)\end{array}$ \\
\hline
\end{tabular}

$\mathrm{CI}$ : confidence interval; COVID-19: coronavirus disease; ECEC: early childhood education and care; HCW: healthcare workers; HR: hazard ratio; IQR: interquartile range; PPE: personal protective equipment; SARS-CoV-2: severe acute respiratory syndrome coronavirus 2; US: United States.

a One case included here is also included in the case series by Cai et al. [17].

${ }^{\mathrm{b}}$ Article was translated using Google Translate. 
TABLE 2D

Characteristics of studies on transmission of SARS-CoV-2 by children, 30 December 2019-10 August 2020 ( $\mathrm{n}=25)$

\begin{tabular}{|c|c|c|c|c|c|c|}
\hline \multirow{2}{*}{ Author } & \multirow{2}{*}{ Country } & \multirow{2}{*}{ Study design } & \multirow{2}{*}{ Population setting } & \multicolumn{2}{|l|}{ Demographics } & \multirow{2}{*}{ Primary outcome results } \\
\hline & & & & Age & Sex & \\
\hline $\begin{array}{l}\text { Xu et al. [25] } \\
\text { (preprint at the } \\
\text { time of writing) }\end{array}$ & China & Observational & $\begin{array}{l}419 \text { index patients and their } 595 \\
\text { household secondary infections; } \\
\text { index patient: first case patient } \\
\text { and the only person in the } \\
\text { household who returned home } \\
\text { from Wuhan/other cities in Hubei } \\
\text { Province; } \\
\text { secondary cases: patients who } \\
\text { had no known exposure to virus } \\
\text { sources outside of the family }\end{array}$ & Not reported & Not reported & $\begin{array}{c}\text { No case } 15 \text { years of age or } \\
\text { younger who was infected } \\
\text { by index patient (first case } \\
\text { patient) was reported; } \\
\text { three index patients were } \\
\text { aged under } 18 \text { years and } \\
\text { infected three secondary } \\
\text { cases, one aged } 0-17 \text { years, } \\
\text { one aged } 18-49 \text { years and one } \\
\text { aged }>65 \text { years }\end{array}$ \\
\hline $\begin{array}{l}\text { Zhu et al. [35] } \\
\text { (preprint at the } \\
\text { time of writing) }\end{array}$ & $\begin{array}{c}\text { China, } \\
\text { Singapore, } \\
\text { South Korea, } \\
\text { Japan, and } \\
\text { Iran }\end{array}$ & $\begin{array}{l}\text { Secondary data } \\
\text { analysis of } \\
\text { published data }\end{array}$ & $\begin{array}{l}31 \text { household transmission } \\
\text { clusters, } 94 \text { cases including } 20 \\
\text { paediatric SARS-CoV-2 cases; } \\
\text { Setting: review of published } \\
\text { literature and datasets between } \\
\text { December } 2019 \text { and March } 2020\end{array}$ & $\begin{array}{l}3 \text { months-10 years } \\
\text { old }(n=20)\end{array}$ & $\begin{array}{l}\text { Male }(n=13) \\
\text { Female }(n=7)\end{array}$ & $\begin{array}{c}\text { Confirmed transmission: } \\
\text { cluster with paediatric index } \\
\text { case: } 3 / 31^{a} \text {; } \\
\text { number of secondary cases: } 5 \text {; } \\
\text { cluster with paediatric } \\
\text { index case, assuming that } \\
\text { asymptomatic children are } \\
\text { being mistakenly overlooked } \\
\text { as the index case in familial } \\
\text { clusters: } 6 / 28\end{array}$ \\
\hline \multicolumn{7}{|c|}{ Educational settings-based transmission } \\
\hline $\begin{array}{l}\text { Dub et al. [36] } \\
\text { (preprint at the } \\
\text { time of writing) }\end{array}$ & $\begin{array}{l}\text { Finland } \\
\text { (Helsinki) }\end{array}$ & $\begin{array}{l}\text { Retrospective } \\
\text { cohort study } \\
\text { with nested } \\
\text { household } \\
\text { transmission } \\
\text { study }\end{array}$ & $\begin{array}{l}\text { Two COVID-19 cases (one } \\
\text { paediatric and one staff) in two } \\
\text { schools and } 184 \text { contacts; } \\
\text { paediatric case: one child and } \\
121 \text { close contacts: } 103 \text { school } \\
\text { contacts ( } 96 \text { pupils from four } \\
\text { classes and eight school staff) } \\
\text { and } 18 \text { sports training contacts } \\
\text { (16 children and two adults); } \\
\text { adult case: one staff member } \\
\text { and } 63 \text { exposed persons: } 52 \\
\text { pupils (two classes) and } 11 \text { staff } \\
\text { members; close contacts: less } \\
\text { than } 2 \text { m, for at least } 15 \text { minutes; } \\
\text { household transmission study: } \\
\text { contacts of secondary cases } \\
\text { identified from adult case: } \\
\text { ( } n=33 \text { ); } \\
\text { close household contacts } \\
\text { ( } n=20 \text { ); regular household } \\
\text { contacts ( } n=9 \text { ); extended } \\
\text { household contacts ( } n=4)\end{array}$ & 12 years old & Not reported & $\begin{array}{c}\text { Transmission: } \\
\text { transmission from child } \\
(89 / 121 \text { contacts tested, } \\
\text { nasopharyngeal and serum } \\
\text { specimens) }(n=0) ; \\
\text { transmission from staff } \\
(42 / 52 \text { contacts tested, serum } \\
\text { specimens): } \\
\text { staff to child }(n=7) ; \\
\text { staff to staff }(n=1) ; \\
\text { household transmission study } \\
\text { (seven cases and } 29 \text { contacts, } \\
\text { serum specimens); } \\
\text { possible transmission from } \\
\text { child to contact }(n=2) ; \\
\text { number of secondary cases: } 5\end{array}$ \\
\hline Heavy et al. [37] & Ireland & Case series & $\begin{array}{l}\text { Six COVID-19 cases (three } \\
\text { paediatric and three adult) with } \\
\text { a history of school attendance } \\
\text { and } 1,155 \text { contacts ( } 1,025 \text { school } \\
\text { contacts, } 130 \text { other settings); } \\
\text { paediatric cases: one primary } \\
\text { school, two secondary school } \\
\text { (905 school contacts, } 84 \text { other) }\end{array}$ & $\begin{array}{l}10-15 \text { years old } \\
(n=3)\end{array}$ & Not reported & $\begin{array}{l}\text { Confirmed transmission from } \\
\text { infected child }(n=0)\end{array}$ \\
\hline
\end{tabular}

$\mathrm{CI}$ : confidence interval; COVID-19: coronavirus disease; ECEC: early childhood education and care; HCW: healthcare workers; HR: hazard ratio; IQR: interquartile range; PPE: personal protective equipment; SARS-CoV-2: severe acute respiratory syndrome coronavirus 2; US: United States.

${ }^{\text {a }}$ One case included here is also included in the case series by Cai et al. [17].

${ }^{\mathrm{b}}$ Article was translated using Google Translate. 
TABLE 2E

Characteristics of studies on transmission of SARS-CoV-2 by children, 30 December 2019-10 August 2020 ( $\mathrm{n}=25$ )

\begin{tabular}{|c|c|c|c|c|c|c|}
\hline \multirow{2}{*}{ Author } & \multirow{2}{*}{ Country } & \multirow{2}{*}{ Study design } & \multirow{2}{*}{ Population setting } & \multicolumn{2}{|l|}{ Demographics } & \multirow{2}{*}{ Primary outcome results } \\
\hline & & & & Age & Sex & \\
\hline $\begin{array}{l}\text { Macartney et al. } \\
\text { [38] }\end{array}$ & $\begin{array}{c}\text { Australia } \\
\text { (New South } \\
\text { Wales) }\end{array}$ & $\begin{array}{l}\text { Prospective } \\
\text { cohort study }\end{array}$ & $\begin{array}{l}27 \text { COVID-19 cases ( } 12 \text { children, } \\
15 \text { staff) and } 1,448 \text { ( } 1,185 \text { student } \\
\text { contacts, } 263 \text { staff contacts) } \\
\text { close contacts from } 15 \text { schools } \\
\text { (10 high schools, five primary } \\
\text { schools) and } 10 \text { ECEC settings; } \\
633 / 1,448 \text { ( } 43.7 \% \text { ) had nucleic } \\
\text { acid testing, or antibody testing; } \\
\text { index case: first identified } \\
\text { laboratory-confirmed case } \\
\text { who attended the facility while } \\
\text { infectious; } \\
\text { primary case: initial infectious } \\
\text { case or cases in that setting, and } \\
\text { might or might not have been the } \\
\text { index case; } \\
\text { secondary case: close contact } \\
\text { with SARS-CoV-2 infection } \\
\text { (detected through nucleic acid } \\
\text { testing or serological testing, } \\
\text { or both), which was considered } \\
\text { likely to have occurred via } \\
\text { transmission in that educational } \\
\text { setting; } \\
\text { close contact: a person who had } \\
\text { been in face-to-face contact for } \\
\text { at least } 15 \text { min or in the same } \\
\text { room for two hours with a case } \\
\text { while infectious; } \\
\text { high schools } \\
\text { so primary cases (three children, } \\
\text { seven staff) with } 534 \text { close } \\
\text { contacts ( } 406 \text { children, } 128 \text { staff) } \\
\text { four staff) with } 696 \text { close } \\
\text { contacts ( } 600 \text { students, } 96 \\
\text { staff); primary schools } \\
\text { five primary cases (one student, } \\
\text { four staff) with } 218 \text { close } \\
\text { contacts ( } 179 \text { students, } 39 \text { staff); } \\
\text { of }\end{array}$ & $\begin{array}{l}\text { High schools } \\
(n=8): \text { median } 15 \\
\text { (range: } 14-16) ; \\
\text { primary schools } \\
(n=1): 10 \\
\text { ECEC }(n=3): \\
\text { median } 2 \text { (range: } \\
2-3)\end{array}$ & $\begin{array}{l}\text { High schools } \\
(n=8) \text { : male } \\
(n=5) ; \text { female } \\
(n=3) ; \\
\text { primary } \\
\text { schools }(n=1) \text { : } \\
\text { female }(n=1) ; \\
\text { ECEC }(n=3) \text { : } \\
\text { male }(n=1) ; \\
\text { female }(n=2)\end{array}$ & $\begin{array}{c}\text { Positive cases: } \\
18 \text { secondary cases: } 10 \\
\text { children, eight staff in } 4 / 25 \\
\text { settings; } \\
\text { cases in schools }(\mathrm{n}=5) \text { : } \\
\text { two secondary schools with } \\
\text { two children and one staff } \\
\text { case (child source); } \\
\text { one primary school with one } \\
\text { child case and one staff case } \\
\text { (adult source); } \\
\text { one ECEC setting ( }=13 \\
\text { cases): seven children, six } \\
\text { staff (adult source); } \\
\text { confirmed transmission } \\
\text { all settings: child case to } \\
\text { child contacts: } 2 / 649 ; \\
\text { all settings: child case to staff } \\
\text { contacts: } 1 / 103 ; \\
\text { all settings, staff contacts to } \\
\text { child case: } 8 / 536 ; \\
\text { all settings, staff contacts to } \\
\text { staff case: } 7 / 160\end{array}$ \\
\hline $\begin{array}{l}\text { Stein-Zamir et al. } \\
\text { [39] }\end{array}$ & Israel & Case series & $\begin{array}{l}\text { Two student COVID-19 cases } \\
\text { in one high school (not } \\
\text { epidemiologically linked) and } \\
1,312 \text { contacts ( } 1,161 \text { student, } \\
151 \text { staff) }\end{array}$ & Not reported & Not reported & $\begin{array}{c}\text { Possible transmission: } \\
\text { positive cases }(\mathrm{n}=178) ; \\
\text { students: } 153 / 1,161(13.2 \%) ; \\
\text { staff: } 25 / 151(16.6 \%) ; \\
\text { COVID-19 rates were higher } \\
\text { in junior grades }(7-9) \text { than in } \\
\text { high grades }(10-12) ; \\
\text { additionally, } 87 \text { confirmed } \\
\text { cases among close contacts } \\
\text { (siblings, recreational } \\
\text { contacts and parents of } \\
\text { students; family members of } \\
\text { school staff) of the school's } \\
\text { cases }\end{array}$ \\
\hline
\end{tabular}

$\mathrm{CI}$ : confidence interval; COVID-19: coronavirus disease; ECEC: early childhood education and care; HCW: healthcare workers; HR: hazard ratio; IQR: interquartile range; PPE: personal protective equipment; SARS-CoV-2: severe acute respiratory syndrome coronavirus 2; US: United States.

a One case included here is also included in the case series by Cai et al. [17].

${ }^{\mathrm{b}}$ Article was translated using Google Translate. 


\begin{tabular}{|c|c|c|c|c|c|c|}
\hline \multirow{2}{*}{ Author } & \multirow{2}{*}{ Country } & \multirow{2}{*}{ Study design } & \multirow{2}{*}{ Population setting } & \multicolumn{2}{|l|}{ Demographics } & \multirow{2}{*}{ Primary outcome results } \\
\hline & & & & Age & Sex & \\
\hline $\begin{array}{l}\text { Yoon et al. [40] } \\
\text { (preprint at the } \\
\text { time of writing) }\end{array}$ & South Korea & $\begin{array}{c}\text { Secondary } \\
\text { analysis of } \\
\text { press release } \\
\text { data }\end{array}$ & $\begin{array}{l}45 \text { paediatric cases in } 40 \text { schools } \\
\text { and kindergartens ( } 12 \text { high } \\
\text { schools, eight middle schools, } \\
15 \text { elementary schools, five } \\
\text { kindergartens) and at least } \\
10,903 \text { contacts }\end{array}$ & $\begin{array}{l}4-5 \text { years old } \\
(n=5) ; \\
6-12 \text { years old } \\
(n=19) ; \\
13-15 \text { years old } \\
(n=8) ; \\
16-18 \text { years old } \\
(n=13)\end{array}$ & Not reported & $\begin{array}{l}\text { Confirmed transmission from } \\
\text { infected child }(\mathrm{n}=1) \text {; } \\
\text { number of secondary cases } \\
\qquad(\mathrm{n}=2) \text {; } \\
\text { 11 year old child in elementary } \\
\text { school transmitted the } \\
\text { SARS-CoV-2 virus to two other } \\
\text { children }\end{array}$ \\
\hline Yung et al. [45] & Singapore & Case series & $\begin{array}{l}\text { Three SARS-CoV-2 cases (two } \\
\text { paediatric and one adult) who } \\
\text { attended two preschools and } \\
\text { one secondary school and } 119 \\
\text { contacts ( } 42 \text { from paediatric } \\
\text { cases, } 93 \text { from adult case); } \\
\text { close contacts (e.g. students } \\
\text { from the same class) were } \\
\text { placed under quarantine; } \\
\text { non-close contacts were not } \\
\text { quarantined and continued with } \\
\text { classes SARS-CoV-2-positive } \\
\text { from contact tracing following } \\
\text { their exposures to adult family } \\
\text { household members who were } \\
\text { part of a community cluster }\end{array}$ & $\begin{array}{l}12 \text { years old and } 5 \\
\text { years old }\end{array}$ & Not reported & $\begin{array}{l}\text { Confirmed transmission from } \\
\text { infected child }(n=0)\end{array}$ \\
\hline
\end{tabular}

CI: confidence interval; COVID-19: coronavirus disease; ECEC: early childhood education and care; HCW: healthcare workers; HR: hazard ratio; IQR: interquartile range; PPE: personal protective equipment; SARS-CoV-2: severe acute respiratory syndrome coronavirus 2; US: United States.

a One case included here is also included in the case series by Cai et al. [17].

${ }^{\mathrm{b}}$ Article was translated using Google Translate.

Two case reports also described transmission of SARSCoV-2 by children in this age group [22,28]. A COVID-19 outbreak in a paediatric ward in South Korea was concluded to be a case of transmission from a 9-year-old child to an adult [28]. From 1,152 close and casual contacts tested, including the index patient's parents and healthcare workers, one contact had a positive SARSCoV-2 PCR result. In a case report on a 7-year-old girl positive for SARS-CoV-2, Lin et al. [22], concluded that the girl infected her father. In both these cases, it is plausible that the source of infection was household or community transmission.

Two case series reported no transmission from children in this age group. In a case series of three paediatric COVID-19 cases (aged 4-8 years) placed in isolation in a hospital in Thailand, one case was isolated with a grandfather co-infected with SARS-CoV-2, while two cases were isolated with their healthy caregivers [26]. Both healthy caregivers tested SARS-CoV-2- negative based on PCR tests of nasopharyngeal and throat swabs for the duration of the isolation, with no symptoms up to 14 days post discharge. Danis et al. [19] investigated a cluster of COVID-19 cases in the French Alps where a 9 -year-old child with COVID-19, co-infected with other respiratory viruses (picornavirus and influenza $A$ ), visited three schools (duration of visits was not reported) and attended one ski class while symptomatic. From 172 contacts, no additional case of COVID-19 linked to the 9-year-old was identified.

\section{Teenagers}

Two studies specifically included teenagers. In one case report, no transmission from a SARS-CoV-2-positive 17-year-old to medical staff was documented following orthopaedic surgery [29]. An outbreak of SARS-CoV-2 at an overnight camp in Georgia, US, was detected after a teenage staff member (age not reported) tested positive for SARS-CoV-2 [27]. In total, test results for 344 of 597 camp attendees (aged 6-59 years) were available and $76 \%$ (260/344) were positive for SARSCoV-2. Camp attendees engaged in a variety of indoor and outdoor activities (e.g. singing and cheering) and adherence to precautionary measures including physical distancing in cabins and use of face masks was not reported. As the authors highlight, given the increasing incidence of COVID-19 in Georgia at the time, household or community transmission cannot be ruled out for these cases, however, it is not clear whether these factors alone explain the high positivity rate observed [27]. 
TABLE 3

Characteristics of studies on transmission modelling of SARS-CoV-2 by children, 30 December 2019-10 August 2020 ( $\mathrm{n}=3$ )

\begin{tabular}{|c|c|c|c|c|c|c|}
\hline \multirow{2}{*}{ Author } & \multirow{2}{*}{ Country } & \multirow{2}{*}{ Study design } & \multirow{2}{*}{$\begin{array}{c}\text { Data source and model } \\
\text { parameters }\end{array}$} & \multicolumn{2}{|c|}{ Demographics } & \multirow{2}{*}{ Results } \\
\hline & & & & Age & Sex & \\
\hline $\begin{array}{l}\text { Dattner } \\
\text { et al. [43] } \\
\text { (preprint at } \\
\text { the time of } \\
\text { writing) }\end{array}$ & Israel & Modelling study & $\begin{array}{c}637 \text { households comprising } 3,353 \\
\text { people in Bnei Brak; } \\
\text { household size ranged from two to } \\
\text { over 10; } \\
\text { household inclusion criteria: at least } \\
\text { two members, with all household } \\
\text { members tested and at least one } \\
\text { with PCR-confirmed COVID-19; } \\
\text { model parameters data sources: } \\
\text { discrete stochastic dynamic model } \\
\text { with based on surveillance data, } \\
\text { parameter estimates obtained by a } \\
\text { maximum likelihood method, where } \\
\text { the likelihood function is computed } \\
\text { based on the stochastic model via } \\
\text { simulations }\end{array}$ & $\begin{array}{l}\text { o-19 years old }(\mathrm{n}= \\
1,544) ; \\
\text { over 20years old } \\
\text { ( } \mathrm{n} 1,809) ; \\
\text { clinical } \\
\text { characteristics: } \\
\text { PCR-confirmed } \\
\text { COVID-19 } \\
\text { 1,510/3,353 (45\%) } \\
\text { 0-19 years old } \\
512 / 1,544(33 \%) ; \\
\text { over } 20 \text { years old } \\
\text { 998/1,809 }(55 \%) ;\end{array}$ & $\begin{array}{l}\text { Not } \\
\text { reported }\end{array}$ & $\begin{array}{l}\text { Transmission modelling: children, } \\
\text { when infected, are somewhat less } \\
\text { prone to infect others compared } \\
\text { with adults, although the result } \\
\text { is not statistically significant; the } \\
\text { infectivity of children is estimated } \\
\text { to be } \mathrm{HR}: 85 \%(95 \% \mathrm{Cl}: 65-110) \\
\text { relative to that of adults }\end{array}$ \\
\hline $\begin{array}{l}\text { James et } \\
\text { al. [44] } \\
\text { (preprint at } \\
\text { the time of } \\
\text { writing) }\end{array}$ & $\begin{array}{c}\text { New } \\
\text { Zealand }\end{array}$ & Modelling study & $\begin{array}{c}\text { Confirmed and probable COVID-19 } \\
\text { cases }(n=1,499) \text {, domestic cases } \\
(n=924)(62 \%) \text {; imported cases } \\
(n=575)(38 \%) \text { from a completed } \\
\text { outbreak; } \\
\text { clinical characteristics: } \\
\text { not reported; model parameters data } \\
\text { sources: comprehensive dataset from } \\
\text { a completed outbreak; } \\
\text { reconstructed multiple instances of } \\
\text { the transmission tree using a Monte- } \\
\text { Carlo technique for cases missing } \\
\text { potential index case or where there } \\
\text { were multiple potential index cases }\end{array}$ & $\begin{array}{l}\text { Under } 10 \text { years old } \\
(n=35) ; \\
0-65 \text { years old } \\
(n=1,261) ; \text { over } 65 \\
\text { years old }(n=172)\end{array}$ & $\begin{array}{l}\text { Not } \\
\text { reported }\end{array}$ & $\begin{array}{l}\text { Transmission: } \\
\text { children infected fewer people on } \\
\text { average and had a lower secondary } \\
\text { attack rate compared with adults } \\
\text { and the elderly; } \\
\text { expected number of secondary } \\
\text { infections caused by age group at } \\
\text { no alert level (pre } 25 \text { March } 2020 \text { ); } \\
\text { under } 10 \text { years old: } 0.87 \text {; } \\
0-65 \text { years old: } 1.49 \text {; } \\
\text { over } 65 \text { years old: } 1.51 \text {; } \\
\text { expected number of secondary } \\
\text { infections caused by age group at } \\
\text { alert level } 4 \text { (post } 25 \text { March } 2020 \text { ); } \\
\text { under } 10 \text { years: }<1 \\
0-65 \text { years: }<1 \\
\text { over } 65 \text { years: }>1\end{array}$ \\
\hline $\begin{array}{l}\text { Zhao et } \\
\text { al. [42] } \\
\text { (preprint at } \\
\text { the time of } \\
\text { writing) }\end{array}$ & China & $\begin{array}{l}\text { Mathematical } \\
\text { modelling }\end{array}$ & $\begin{array}{l}29 \text { COVID-19 cases; } 10 \text { with history of } \\
\text { exposure to Huanan seafood market; } \\
19 \text { without exposure; } \\
\text { model parameters data sources: } \\
\text { age group proportions, birth rate } \\
\text { and death rate (Wuhan statistical } \\
\text { yearbook); other parameters: } \\
\text { literature }\end{array}$ & Not reported & $\begin{array}{l}\text { Not } \\
\text { reported }\end{array}$ & $\begin{array}{c}\text { Model with four-age groups: } \\
\text { highest transmissibility } \\
\text { occurred between the age groups } \\
15-44 \text { years and } 45-64 \text { years, } \\
\text { among those } 65 \text { years and older, or } \\
\text { from } 45-64 \text { years to } 65 \text { years and } \\
\text { older; } \\
\text { lowest transmissibility occurred } \\
\text { from age group 0-14 years to } \\
\text { 15-44 years, or from } 45-64 \text { years } \\
\text { to } \leq 14 \text { years; } \\
\text { model with five-age groups: } \\
\text { highest transmissibility } \\
\text { occurred between age group } 25-59 \\
\text { years and } \geq 60 \text { years, or among } \\
25-59 \text { years; } \\
\text { lowest transmissibility occurred } \\
\text { from age group } 15-24 \text { years to } \\
25-59 \text { years, or from age group } 0-5 \\
\text { years to } 6-14 \text { years, or, to } 15-24 \\
\text { years }\end{array}$ \\
\hline
\end{tabular}

COVID-19: coronavirus disease; SARS-CoV-2: severe acute respiratory syndrome coronavirus 2. 
Methodological quality of included studies on transmission of SARS-CoV-2 by children, 30 December 2019-10 August 2020 $(\mathrm{n}=28)$

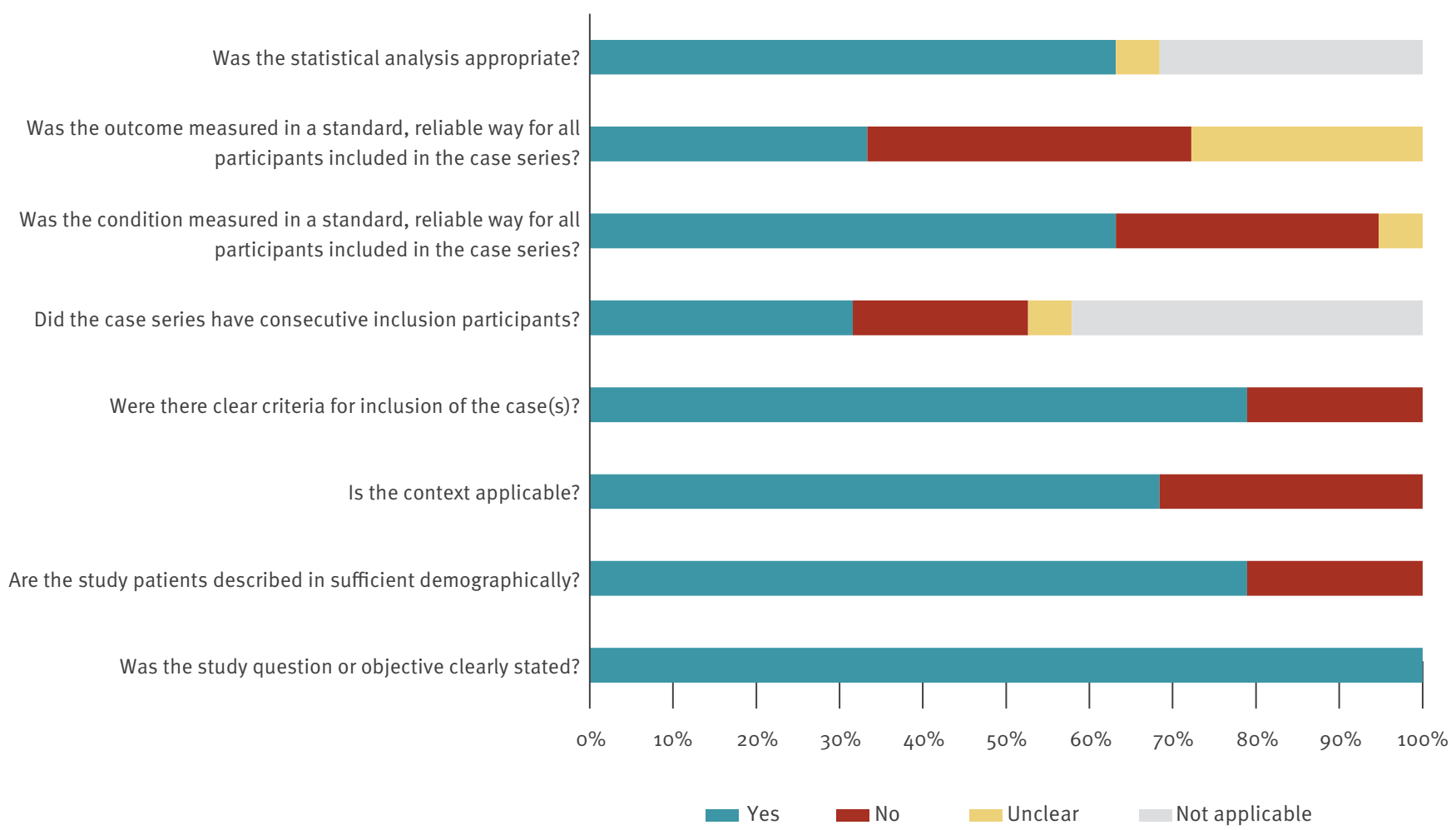

SARS-CoV-2: severe acute respiratory syndrome coronavirus 2.

\section{Age unspecified}

The remaining seven studies included children across a wide range of ages. Children younger than 16 years old were reported to be the index case in $8 \%$ of households (3/39, ages not specified) in Switzerland [24] and Israel (1/13 families, 14-year-old) [33]. Children aged 0-17 years were reported to be the index case in four of 15 households in Chicago, US [32], with two of the four household transmission clusters reported as child-tochild and two as child-to-adult [32]. In these studies, families were generally asked to self-report whether they developed symptoms before, after, or at the same time as the child case. Two of the three analyses of surveillance data documented transmission from children $[31,34]$. An analysis of 732 infector-infectee pairs conducted by the Dutch National Institute for Public Health and the Environment noted that COVID-19 was primarily spread between persons of approximately the same age [34]. Within the dataset, 23 of 31 cases aged under 20 years were infected in the home. Two of these 23 cases were categorised as child-to-child transmission although in one instance the source case was in the age group 15-20 years [34].

A study of public health surveillance data of confirmed cases and contacts in the high incidence setting of India, which included 42,618 children aged $0-17$ years with 57,415 contacts, also found the highest probability of transmission within cases and contacts of a similar age [31]. A case series of 74 children with COVID-19 admitted to two hospitals in China reported no evidence that the virus was transmitted from these 74 children to others, although there is limited reporting on how this information was ascertained [18]. An analysis of local heath commissions' public disclosures data in China [25], based on 419 index patients and their 595 household secondary infections, reported no cases of infection by an index patient 15 years of age or younger. In a linked publication, the authors estimate that the hazard of being infected within households was higher for those aged under 18 years and those aged over 65 years, whereas the hazard of being infected outside of households was higher for those aged 18-64 years [46].

In summary, across these 19 household and close contact studies (where data was available), from a total of 42,926 reported children there were 42,639 cases where a child was considered to be the index case and 4,381 infected contacts (predominantly family members) were identified (Table 4). However, over $90 \%$ of these were from one study in a high incidence setting [31]. Where the specific age of index case was reported, transmission tended to occur from older children rather than from infants and young children. The total number of contacts could not be estimated and the majority of studies (10/19) did not report number of cases followed up. Where reported, contact numbers ranged from 1 to $57,415[19,24,26-29,31,33]$ and the majority of contacts in those studies were not infected. 
Educational setting transmission

Six studies assessed transmission within educational settings [36-41]. All six studies included children in secondary school settings [36-41], while three also included children in preschool settings $[38,40,41]$ and three additionally included children in primary school settings $[37,38,40]$. Three of six studies reported transmission of SARS-CoV-2 by children [38-40].

A study from New South Wales, Australia examined SARS-CoV-2 transmission in 25 educational settings, 15 primary and secondary schools, and 10 early childhood education and care settings (ECEC) over a 10-week period (25 January 2020-9 April 2020 with distance learning encouraged statewide in March) [38]. They identified 27 confirmed cases, 12 children and 15 staff members who attended school or ECEC settings while considered infectious, defined as $24 \mathrm{~h}$ before symptom onset, and 1,448 close contacts $(1,185$ students and 263 staff). In total, 633 of 1,448 close contacts had nucleic acid or antibody testing, with 18 secondary cases (10 children and eight adults) identified. Three secondary cases (one adult, two children) were linked to transmission from three children of secondary school age, resulting in an overall secondary attack rate of $0.3 \%$ and a secondary attack rate for child to staff member of $1.0 \%$.

Based on analysis of data reported in press releases on 45 paediatric patients with COVID-19 in 40 schools and kindergartens, and 10,903 tested contacts by the Korean Centers for Disease Control and Prevention, Yoon et al. [40] reported one case where an 11-year-old child transmitted the virus to two other children. One child was infected in the same classroom and the other child was infected at the same gym outside the school setting. In Israel, a large outbreak was reported 10 days after schools reopened [39]. Following diagnosis of two unrelated cases, the entire school community i.e. 151 staff members and 1,161 students, was tested; 178 (25 staff $16.6 \%), 153$ students (13.2\%) tested positive for SARS-CoV-2. During this time, there was an extreme heat wave, with school attendees exempt from mask wearing, the air conditioning was continuously on and physical distancing could not be observed in crowded classrooms, which may have contributed to the spread of the virus.

The remaining three studies in educational settings reported no transmission of SARS-CoV-2 by children. An analysis of Irish notifications of SARS-CoV-2 in the school setting, before universal school closure on 12 March 2020 and introduction of public health measures, found no transmission from children [37]. Three paediatric cases (all aged 10-15 years) and three adult cases of COVID-19 were identified. The three paediatric cases had a total of 822 child and 83 adult contacts within the school setting. Contacts were exposed at school in the classroom and during school-related activities such as sports lessons, music lessons and choir practice. No additional cases were identified during the follow-up period of 14 days from last contact with the index case. However, only contacts who developed symptoms were referred for testing, thus asymptomatic secondary cases were not captured. Similarly, a report of SARS-CoV-2 transmission among children in two preschools and one secondary school in Singapore found no transmission from children [45]. At the time, schools in Singapore were not routinely closed, but targeted public health measures including deep cleaning of the schools and suspension of extracurricular activities were implemented. From a child index case aged 5 years in one preschool, a total of 34 preschool student contacts developed symptoms post-exposure and were tested for SARS-CoV-2, but SARS-CoV-2 was not detected. From a secondary school child index case aged 12 years, a total of eight students developed symptoms and were screened for SARS-CoV-2 during the incubation period, all of whom tested negative [45]. A report of SARS-CoV-2 transmission among children in two schools in Finland also found no transmission from children [36]. Exposure in school because of a 12 -year-old index case who attended with mild symptoms led to no further cases among 89 of 121 close contacts tested.

In summary, across these six studies, there were 65 cases where a student was considered to be the index case and 183 additional infected contacts were identified from 14,003 close contacts (Table 5). There was no transmission from a child reported in the pre-school setting and one case where a primary school child was considered to be the index case (with two additional infected contacts). In the majority of cases, transmission from a child was reported in the secondary school setting. There were 35 secondary school index cases with 183 additional infected contacts, although the majority of these (97\%) were from one study [39]. However, household or community transmission cannot be ruled out for these cases.

\section{Transmission modelling}

All three modelling studies estimated lower transmission in children compared with adults [42-44]. In a mathematical modelling study (using an adapted version of the of Susceptible-Exposed-Infectious-Recovered (SEIR) model) estimating age-specific transmissibility of SARS-CoV-2, Zhao et al. [42] concluded that SARSCoV-2 had high transmissibility among adults aged 25 years or older, but low transmissibility among children younger than 14 years of age. The model fit was based on data from 29 cases, 10 of whom had exposure to the Huanan seafood market. Dattner et al. [43] fitted a dynamic stochastic age-of-infection model to estimate household SARS-CoV-2 transmission in Bnei Brak, Israel. Data were collected from 637 households $(n=3,353)$ of at least two members, with all household members tested and at least one with PCR-confirmed COVID-19. Transmission was estimated to be lower in children and young people (aged 0-19 years), compared with adults (aged 20 years or older), although this difference was not statistically significant (hazard 
TABLE 4

Reported SARS-CoV-2 transmission by children in households and close contact transmission studies, 30 December 2019-10 August $2020(n=19)$

\begin{tabular}{|c|c|c|c|c|}
\hline Authors & $\begin{array}{l}\text { Number of children } \\
\text { included }\end{array}$ & $\begin{array}{l}\text { Number of paediatric } \\
\text { index cases }\end{array}$ & $\begin{array}{l}\text { Number of infected } \\
\text { contacts }\end{array}$ & $\begin{array}{l}\text { Number of contacts } \\
\text { followed up }\end{array}$ \\
\hline \multicolumn{5}{|c|}{ Children under 2 years of age } \\
\hline Canarutto et al. [20] & 1 & o & 0 & Not reported \\
\hline Le et al. [21] & 1 & 0 & 0 & Not reported \\
\hline Nassih et al. [30] & 1 & 0 & 0 & 1 \\
\hline Qui et al. [23] & 1 & o & 0 & Not reported \\
\hline \multicolumn{5}{|l|}{ Children aged $0-10$ years } \\
\hline Cai et al. [17] & 10 & 1 & 2 & Not reported \\
\hline Danis et al. [19] & 1 & o & o & 172 \\
\hline Jung et al. [28] & 1 & 1 & 1 & 1,206 \\
\hline Lin et al. [22] & 1 & 1 & 1 & Not reported \\
\hline Wongsawat et al. [26] & 3 & o & 0 & 2 \\
\hline Zhu et al. [35] & 20 & 3 & 5 & Not reported \\
\hline \multicolumn{5}{|l|}{ Teenagers } \\
\hline Lucar et al. [29] & 1 & 1 & 0 & 11 \\
\hline Szablewski et al. [27] & 1 & 1 & 260 & 597 \\
\hline \multicolumn{5}{|l|}{ Age unspecified } \\
\hline Laxminarayan et al. [31] & 42,618 & 42,618 & 4,110 & 57,415 \\
\hline Mannheim et al. [32] & 64 & 4 & Not reported & Not reported \\
\hline Posfay-Barbe et al. [24] & 39 & 3 & 4 & 111 \\
\hline Somekh et al. [33] & 58 & 1 & Not reported & 94 \\
\hline Van Der Hoek et al. [34] & 31 & 2 & $2^{\mathrm{b}}$ & Not reported \\
\hline Wu et al. [18] & 74 & 0 & 0 & Not reported \\
\hline Xu et al. [25] & Unclear $^{c}$ & 3 & 3 & Not reported \\
\hline Total & 42,926 & 42,639 & 4,381 & Not estimable \\
\hline
\end{tabular}

SARS-CoV-2: severe acute respiratory syndrome coronavirus 2.

+ Some cases included may be double counted across studies.

${ }^{\mathrm{b}}$ The source in one of these cases was aged 15-20 years.

c Unclear how many children included overall.

ratio: $85 \%$; $95 \%$ confidence interval: $65-110)$. James et al. [44], analysed the transmission dynamics of COVID19 using a comprehensive dataset for a completed outbreak (at the time) in New Zealand. They estimated that although children younger than 10 years old were equally likely to infect at least one person, they contributed less to the spread of SARS-CoV-2 than other age groups, infecting fewer people on average and having a lower secondary attack rate.

\section{Discussion}

The evidence in this review highlights that child-tochild and child-to-adult transmission of SARS-CoV-2 can occur. However, unlike in other viral epidemics, for example influenza [47], during the early stages of the COVID-19 pandemic, children did not appear to be key transmitters of SARS-CoV-2 with reported transmission from children being low. Studies published subsequent to this review have highlighted that the proportion of paediatric COVID-19 cases has risen as overall transmission has increased [5]. The majority of included studies have looked at household transmission related to known cases and it was often unclear who the index patient was. These results are consistent with similar previous reviews in the area $[48,49]$. In these studies, children aged 10 years and older may spread the virus more easily to family members than younger children and might even spread it as easily as adults [50]. Although not all studies presented a breakdown of children's age, our results are consistent with this finding, with more cases of transmission linked to older children than younger children. However, these results should be interpreted in the context of the limitations of included studies.

Ten of the 19 included studies on household and close contact transmission reported child-to-child or child-to-adult transmission. Overall, from 19 studies, with a total of 42,926 reported children, 42,639 cases where a child was considered to be the index case and 4,381 infected contacts, predominantly family members, were identified. Four household transmission studies reported that children were the index case in $8 \%$ to $26 \%$ of households [24,32,33,35]. Zhu et al. [35] reported that even if the assumption was made that asymptomatic children were the index case in all 
TABLE 5

Reported SARS-CoV-2 transmission by children in school-based studies, 30 December 2019-10 August 2020 ( $\mathrm{n}=6$ )

\begin{tabular}{|c|c|c|c|}
\hline Author & $\begin{array}{c}\text { Number of paediatric index } \\
\text { cases }\end{array}$ & $\begin{array}{l}\text { Number of contacts } \\
\text { followed up }\end{array}$ & $\begin{array}{c}\text { Number of infected contacts from paediatric } \\
\text { index cases }\end{array}$ \\
\hline \multicolumn{4}{|c|}{ Pre-school (aged 0-5 years) } \\
\hline Macartney et al. [38] & 3 & 122 & 0 \\
\hline Yoon et al. [40] & 5 & 670 & 0 \\
\hline Yung et al. [41] & 1 & 34 & 0 \\
\hline \multicolumn{4}{|c|}{ Primary school (aged 5-11 years) } \\
\hline Macartney et al. [38] & 1 & 45 & 0 \\
\hline Heavy et al. [37] & 1 & $905^{\mathrm{a}}$ & 0 \\
\hline Yoon et al. [40] & 19 & $3,524^{b}$ & 2 \\
\hline \multicolumn{4}{|c|}{ Secondary school (aged 12 years or older) } \\
\hline Dub et al. [36] & 1 & 89 & 0 \\
\hline Heavy et al. [37] & 2 & $905^{\mathrm{a}}$ & 0 \\
\hline Macartney et al. [38] & 8 & 586 & 3 \\
\hline Stein-Zamir et al. [39] & 2 & 1,312 & 178 \\
\hline Yoon et al. [40] & 21 & $6,709^{b}$ & 0 \\
\hline Yung et al. [41] & 1 & 8 & 0 \\
\hline Total & 65 & 14,003 & 183 \\
\hline
\end{tabular}

SARS-CoV-2: severe acute respiratory syndrome coronavirus 2.

a Number of contacts followed up by age of index case is not presented; number of contacts only counted once in overall total in this table.

b 1,071 individuals were tested because of exposures from two brothers in the same family, one from a primary school and one from a secondary school setting. These 1,071 individuals have only been added to the primary school total.

familial clusters, it would still only produce a situation where children accounted for roughly $20 \%$ of household cluster transmissions. All three studies examining large scale surveillance data reported transmission from children at varying levels [27,31,34]. Two of these studies highlight that SARS-CoV-2 is primarily spread between persons of approximately the same age and the probability for contacts to have a positive test result is not clearly associated with their age [34]. Across the remaining case series and case reports, transmission was variable with a lack of information and inconsistencies of reporting within the manuscripts. In at least three studies that suggested transmission from children to other family members occurred $[17,22,28]$, it is also feasible that the virus was transmitted from the parents or adults to the children. To date, published studies indicate that children are mostly infected by family members in the home [51-55]. Many SARS-CoV-2 clusters have been linked to a wide range of mostly indoor settings such as households and few reports came from schools [56]. This is perhaps unsurprising as many of the included studies were conducted in the context of strict physical distancing policies including school closures. Analysis of social contact and agemixing patterns in China during the outbreak period, where strict physical distancing policies were in place, highlights that typical features of age-mixing (school contacts, workplace contacts and so on) decreased and contacts during the outbreak mostly occurred at home with household members $[9,10]$.
Three included case reports $[20,28,29]$ reported no transmission from children to healthcare workers. One study described the use of PPE consisting of N95 mask, face shield, gowns, and gloves [29], but the other studies did not provide any details. The risk of occupational exposure to SARS-CoV-2 may be lower in paediatric healthcare settings, because of a lower number of COVID-19 patients admitted in this setting and to higher awareness and compliance with protective measures [57].

Following a survey of the experience of 15 European countries related to the role of childcare and school settings in COVID-19 transmission in July 2020, and a review of the literature, the European Centre for Disease Prevention and Control (ECDC) reported few large-scale outbreaks of COVID-19 in schools [7]. The results of our review, which includes one additional new study and one updated study with data beyond the ECDC report, are in line with these findings. Across studies on transmission of SARS-CoV-2 in schools included in our review, there were 66 cases where a student was considered to be the index case and 183 additional infected contacts were identified from 14,513 close contacts. The majority of these cases (178/183) were identified in one study from Israel [39], where precautionary measures, such as mask wearing and physical distancing, were not observed and where household or community transmission cannot be ruled out for the cases. Studies published after the last date of our search, have highlighted that transmission in childcare settings may occur, but at low levels [58-60]. Studies 
from Germany, Italy and England have also reported that outbreaks have been uncommon or small since the reopening of schools began [61-64].

A rapid review on school closure during coronavirus outbreaks, including COVID-19, found limited and conflicting information [65]. The authors cite modelling studies of COVID-19 which predict that school closures alone would prevent $2 \%$ to $4 \%$ of deaths, much less than other physical distancing measures [65]. Subsequent modelling studies also report that school closures alone have limited impact on reducing the burden of COVID-19 [66,67]. The ECDC report also concluded closures of childcare and educational institutions are unlikely to be an effective single control measure for community transmission of COVID-19 [7]. However, school closures may have negative impacts for children including interruption of learning, mental health issues and exacerbation of disparities [7]. A systematic review conducted up to July 2021 also concluded that transmission of SARS-CoV-2 was markedly lower in school compared with household settings, suggesting that household transmission is more important than school transmission, particularly in the early pandemic [68].

All three modelling studies estimated lower transmission in children; however, there were few child index cases included across studies [42-44].

Although studies of real-life transmission remain limited, transmission potential of SARS-CoV-2 by children is influenced by a number of factors other than susceptibility to infection, including potential for exposure to the virus and viral load (i.e., the amount of virus that a child might carry). In relation to exposure to the virus outside of the home, a number of studies looking for evidence of past infection in childcare and school settings have been conducted. While these studies do not present evidence on actual transmission chains from infected children to others, they do characterise the burden of COVID-19 within these settings during the epidemic period. Across five studies, SARS-CoV-2 seroprevalance ranged from $0.7 \%$ to $39 \%$ for pupils and $0.2 \%$ to $43 \%$ for teachers [69-73]. A study in Belgian day care settings, undertaken shortly after the start of the local epidemic and before lockdown commenced, noted that while cold symptoms were common, RT-PCR tests of nasopharyngeal specimens taken from a random sample of children $(n=84)$ attending eight different day care centres were all negative for SARS-CoV-2 [74]. A study from January 2020 to March 2021 found that seropositivity in Israeli children aged $0-15$ years was 1.8-5.5 times higher than COVID-19 incidence rates based on PCR testing [75].

Reliable, large-scale data on transmission from symptomatic and asymptomatic children are lacking. Largescale studies focusing on transmission chains using data collected from contact tracing and case investigations would be needed to determine how children contributed to the spread of SARS-CoV-2. As schools and childcare facilities gradually reopened after summer 2020 worldwide, more data on chains of transmission linked to children outside of the household setting should be available. Serological studies looking for past evidence of infection, and studies assessing viral load in infected children and the relationship between viral load and transmission may also be helpful in understanding the role children play in transmission [76]. While we have adhered to fundamental methodological principles, in keeping with international rapid review methodology guidance $[14,77]$, our study has some limitations. For study designs where no universally accepted quality appraisal tool existed (e.g. case series, modelling studies), a series of questions adapted from related tools were used, as described in the protocol and Supplement but it was not a validated tool [13]. Studies of household transmission which describe presumed contact and transmission, but did not specifically report transmission from child to adult [78] or the age of the index cases [79] were not included in this review. The initial search strategy was used to retrieve research relevant to COVID-19 specifically to address a number of review questions at the same time, therefore a detailed flow diagram was not kept as titles and abstracts were screened against a number of research questions.

\section{Conclusion}

During the early pandemic there was limited and poor quality information on the contribution of children to the transmission of SARS-CoV-2. Few definitive cases of virus transmission from children had been published by the beginning of August 2020, with no clear evidence suggesting a higher rate of transmission from children than adults. The published studies identified, indicated that children were not disproportionately contributing to the household transmission of SARSCoV-2 during the early pandemic, however, further high quality studies would be needed.

\section{Acknowledgements}

The authors would like to thank Executive Assistant Debra Spillane (Health Information and Quality Authority (HIQA)) and Information Specialist Paul Murphy (RCSI University of Medicine and Health Sciences), and acknowledge the support of the Health Technology Assessment and Health Information and Standards Directorates at HIQA.

\section{Conflict of interest}

None declared.

\section{Authors' contributions}

All authors conceived the study protocol. Literature searches were conducted by SA and PGC. Screening, data extraction and quality appraisal were conducted by $B C, K J, S A, K A W$, PB, LD, KOB. BC, MON, PH, SMS, MR drafted the final manuscript which was read and approved by all authors. 


\section{References}

1. Dong E, Du H, Gardner L. An interactive web-based dashboard to track COVID-19 in real time. Lancet Infect Dis. 2020;20(5):533-4. https://doi.org/10.1016/S14733099(20)30120-1 PMID: 32087114

2. Sohrabi C, Alsafi Z, O'Neill N, Khan M, Kerwan A, Al-Jabir A, et al. World Health Organization declares global emergency: A review of the 2019 novel coronavirus (COVID-19). Int J Surg. 2020;76:71-6. https://doi.org/10.1016/j.ijsu.2020.02.034 PMID: 32112977

3. Choi S-H, Kim HW, Kang J-M, Kim DH, Cho EY. Epidemiology and clinical features of coronavirus disease 2019 in children. Clin Exp Pediatr. 2020;63(4):125-32. PMID: 32252139

4. Qiu H, Wu J, Hong L, Luo Y, Song Q, Chen D. Clinical and epidemiological features of 36 children with coronavirus disease 2019 (COVID-19) in Zhejiang, China: an observational cohort study. Lancet Infect Dis. 2020;20(6):689-96. https:// doi.org/10.1016/S1473-3099(20)30198-5 PMID: 32220650

5. Bundle N, Dave N, Pharris A, Spiteri G, Deogan C, Suk JE. COVID-19 trends and severity among symptomatic children aged 0-17 years in 10 European Union countries, 3 August 2020 to 3 October 2021. Euro Surveill. 2021;26(50):2101098. https://doi.org/10.2807/1560-7917.ES.2021.26.50.2101098 PMID: 34915968

6. Centers for Disease Control and Prevention (CDC). COVID Data Tracker. Demographic trends of COVID-19 cases and deaths in the US reported to CDC. Atlanta: CDC; 2020. Available from: https://covid.cdc.gov/covid-data-tracker/\#datatracker-home

7. European Centre for Disease Prevention and Control (ECDC). COVID-19 in children and the role of school settings in COVID-19 transmission. Stockholm: ECDC; 2020. Available from: https://www.ecdc.europa.eu/en/publications-data/ children-and-school-settings-covid-19-transmission

8. Sisk B, Cull W, Harris IM, Rothenburger A, Olson L. National trends of cases of COVID-19 in children based on US state health department data. Pediatrics. 2020;146(6):e2020027425. https://doi.org/10.1542/peds.2020-027425 PMID: 32994175

9. Liu Y, Gu Z, Xia S, Shi B, Zhou XN, Shi Y, et al. What are the underlying transmission patterns of COVID-19 outbreak? An age-specific social contact characterization. EClinicalMedicine. 2020;22:100354. https://doi.org/10.1016/j. eclinm.2020.100354 PMID: 32313879

10. Zhang J, Litvinova M, Liang Y, Wang Y, Wang W, Zhao S, et al. Changes in contact patterns shape the dynamics of the COVID-19 outbreak in China. Science. 2020;368(6498):1481-6. https://doi.org/10.1126/science.abb8001 PMID: 32350060

11. Viner RM, Mytton OT, Bonell C, Melendez-Torres GJ, Ward J, Hudson L, et al. Susceptibility to SARS-CoV-2 infection among children and adolescents compared with adults: a systematic review and meta-analysis. JAMA Pediatr. 2021;175(2):143-56. https://doi.org/10.1001/jamapediatrics.2020.4573 PMID: 32975552

12. Davies NG, Klepac P, Liu Y, Prem K, Jit M, Eggo RM. Agedependent effects in the transmission and control of COVID-19 epidemics. Nat Med. 2020;26(8):1205-11. https://doi. org/10.1038/s41591-020-0962-9 PMID: 32546824

13. Health Information and Quality Authority (HIQA). Protocol for evidence synthesis support - COVID-19. Dublin: HIQA; 2020. Available from: https://www.hiqa.ie/sites/default/ files/2020-04/Protocol-for-evidence-synthesis-support_1-4COVID-19.pdf

14. Garritty C, Gartlehner G, Nussbaumer-Streit B, King VJ, Hame C, Kamel C, et al. Cochrane Rapid Reviews Methods Group offers evidence-informed guidance to conduct rapid reviews. J Clin Epidemiol. 2021;130:13-22. https://doi.org/10.1016/j. jclinepi.2020.10.007 PMID: 33068715

15. Health Information and Quality Authority (HIQA). HIQA publishes COVID-19 evidence summaries to support work of the National Public Health Emergency Team. Dublin: HIQA 2020. Available from: https://www.hiqa.ie/hiqa-news-updates/ hiqa-publishes-covid-19-evidence-summaries-support-worknational-public-health

16. Health Information and Quality Authority (HIQA). Evidence summary of potential for children to contribute to transmission of SARS-CoV-2 21 August 2020. HIQA: Dublin; 2020. Available from: https://www.hiqa.ie/ reports-and-publications/health-technology-assessment/ evidence-summary-spread-covid-19-children

17. Jiehao C, Jin X, Daojiong L, Zhi Y, Lei X, Zhenghai Q, et al. A case series of children with 2019 novel coronavirus infection: clinical and epidemiological features. Clin Infect Dis. 2020;71(6):1547-51. https://doi.org/10.1093/cid/ciaa198 PMID: 32112072

18. Wu Q, Xing Y, Shi L, Li W, Gao Y, Pan S, et al. Coinfection and other clinical characteristics of COVID-19 in children.
Pediatrics. 2020;146(1):e20200961. https://doi.org/10.1542/ peds.2020-0961 PMID: 32376725

19. Danis K, Epaulard O, Bénet T, Gaymard A, Campoy S, Botelho-Nevers E, et al. Cluster of Coronavirus Disease 2019 (COVID-19) in the French Alps, February 2020. Clin Infect Dis. 2020;71(15):825-32. https://doi.org/10.1093/cid/ciaa424 PMID: 32277759

20. Canarutto D, Priolo A, Russo G, Pitea M, Vigone MC, Barera G. COVID-19 infection in a paucisymptomatic infant: Raising the index of suspicion in epidemic settings. Pediatr Pulmonol. 2020;55(6):E4-5. https://doi.org/10.1002/ppul.24754 PMID: 32250049

21. Le HT, Nguyen LV, Tran DM, Do HT, Tran HT, Le YT, et al. The first infant case of COVID-19 acquired from a secondary transmission in Vietnam. Lancet Child Adolesc Health. 2020;4(5):405-6. https://doi.org/10.1016/S23524642(20)30091-2 PMID: 32213326

22. Lin J, Duan J, Tan T, Fu Z, Dai J. The isolation period should be longer: Lesson from a child infected with SARS-CoV-2 in Chongqing, China. Pediatr Pulmonol. 2020;55(6):E6-9. https:// doi.org/10.1002/ppul.24763 PMID: 32243729

23. Qiu L, Jiao R, Zhang A, Chen X, Ning Q, Fang F, et al. A case of critically ill infant of coronavirus disease 2019 with persistent reduction of $t$ lymphocytes. Pediatr Infect Dis J. 2020;39(7):e87-90. https://doi.org/10.1097/ INF.0000000000002720 PMID: 32379199

24. Posfay-Barbe KM, Wagner N, Gauthey M, Moussaoui D, Loevy N, Diana A, et al. COVID-19 in children and the dynamics of infection in families. Pediatrics. 2020;146(2):e20201576. https://doi.org/10.1542/peds.2020-1576 PMID: 32457213

25. Xu X, Liu X, Wu Y. ALI ST. Close contacts and household transmission of SARS-CoV-2 in China: a content analysis based on local Heath Commissions' public disclosures. medRxiv. 2020:2020.03.02.20029868. Preprint.

26. Wongsawat J, Moolasart V, Srikirin P, Srijareonvijit C, Vaivong N, Uttayamakul S, et al. Risk of novel coronavirus 2019 transmission from children to caregivers: A case series. J Paediatr Child Health. 2020;56(6):984-5. https://doi. org/10.1111/jpc.14965 PMID: 32567772

27. Szablewski CM, Chang KT, Brown MM, Chu VT, Yousaf AR, Anyalechi N, et al. SARS-CoV-2 transmission and infection among attendees of an overnight camp - Georgia, June 2020 MMWR Morb Mortal Wkly Rep. 2020;69(31):1023-5. https:// doi.org/10.15585/mmwr.mm6931e1 PMID: 32759921

28. Jung J, Hong MJ, Kim EO, Lee J, Kim MN, Kim SH. Investigation of a nosocomial outbreak of coronavirus disease 2019 in a paediatric ward in South Korea: successful control by early detection and extensive contact tracing with testing. Clin Microbiol Infect. 2020;26(11):1574-5. https://doi.org/10.1016/j. cmi.2020.06.021 PMID: 32593744

29. Lucar J, Navalkele B, Becker BP, Reed CD, Parham J. Health care personnel exposure to a patient with asymptomatic SARS$\mathrm{CoV}_{2}$ infection during a prolonged surgical intervention. Am J Infect Control. 2020;48(8):955-7. https://doi.org/10.1016/j. ajic.2020.05.036 PMID: 32505792

30. Nassih H, El Fakiri K, Sab IA. Absence of evidence of transmission of coronavirus disease 2019 from a young child to mother despite prolonged contact. Indian J Pediatr. 2020;87(9):754. https://doi.org/10.1007/s12098-020-03382-0 PMID: 32535688

31. Laxminarayan R, Wahl B, Dudala SR, Gopal K, Mohan B C, Neelima S, et al. Epidemiology and transmission dynamics of COVID-19 in two Indian states. Science. 2020;370(6517):691-7. https://doi.org/10.1126/science.abd7672 PMID: 33154136

32. Mannheim J, Gretsch S, Layden JE, Fricchione MJ. Characteristics of hospitalized pediatric coronavirus disease 2019 cases in Chicago, Illinois, March-April 2020. J Pediatric Infect Dis Soc. 2020;9(5):519-22. https://doi.org/10.1093/ jpids/piaao70 PMID: 32479632

33. Somekh E, Gleyzer A, Heller E, Lopian M, Kashani-Ligumski $\mathrm{L}$, Czeiger S, et al. The role of children in the dynamics of intra family coronavirus 2019 spread in densely populated area. Pediatr Infect Dis J. 2020;39(8):e202-4. https://doi. org/10.1097/INF.0000000000002783 PMID: 32496407

34. van der Hoek W, Backer JA, Bodewes R, Friesema I, Meijer A, Pijnacker $\mathrm{R}$, et al. De rol van kinderen in de transmissie van SARS-CoV-2. [The role of children in the transmission of SARSCoV-2]. Dutch. Ned Tijdschr Geneeskd. 2020;164(25):D5140. PMID: 32749807

35. Zhu Y, Bloxham CJ, Hulme KD, Sinclair JE, Tong ZWM, Steele $\mathrm{LE}$, et al. Children are unlikely to have been the primary source of household SARS-CoV-2 infections. medRxiv. 2020:2020.03.26.20044826. Preprint.

36. Dub T, Erra E, Hagberg L, Sarvikivi E, Virta C, Jarvinen A, et al. Transmission of SARS-CoV-2 following exposure in school settings: experience from two Helsinki area exposure 
incidents. medRxiv. 2020:2020.07.20.20156018. Preprint https://doi.org/10.1101/2020.07.20.20156018

37. Heavey L, Casey G, Kelly C, Kelly D, McDarby G. No evidence of secondary transmission of COVID-19 from children attending school in Ireland, 2020. Euro Surveill. 2020;25(21):2000903. https://doi.org/10.2807/1560-7917.ES.2020.25.21.2000903 PMID: 32489179

38. Macartney K, Quinn HE, Pillsbury AJ, Koirala A, Deng L, Winkler $\mathrm{N}$, et al. Transmission of SARS-CoV-2 in Australian educational settings: a prospective cohort study. Lancet Child Adolesc Health. 2020;4(11):807-16. https://doi.org/10.1016/S23524642(20)30251-0 PMID: 32758454

39. Stein-Zamir C, Abramson N, Shoob H, Libal E, Bitan M, Cardash $\mathrm{T}$, et al. A large COVID-19 outbreak in a high school 10 days after schools' reopening, Israel, May 2020. Euro Surveill. 2020;25(29):2001352. https://doi.org/10.2807/1560-7917. ES.2020.25.29.2001352 PMID: 32720636

40. Yoon Y, Kim K-R, Park H, Kim S, Kim YJ. Stepwise school opening and an impact on the epidemiology of COVID-19 in the children. J Korean Med Sci. 2020;35(46):e414. https://doi. org/10.3346/jkms.2020.35.e414 PMID: 33258334

41. Yung CF, Kam KQ, Nadua KD, Chong CY, Tan NWH, Li J, et al. Novel coronavirus 2019 transmission risk in educational settings. Clin Infect Dis. 2021;72(6):1055-8. https://doi. org/10.1093/cid/ciaa794 PMID: 32584975

42. Zhao Z, Zhu Y-Z, Xu J-W, Hu Q-Q, Lei Z, Rui J, et al. A mathematical model for estimating the age-specific transmissibility of a novel coronavirus. medRxiv. 2020:2020.03.05.20031849. Preprint . https://doi.org/10.1101/ 2020.03.05.20031849

43. Dattner I, Goldberg Y, Katriel G, Yaari R, Gal N, Miron Y, et al. The role of children in the spread of COVID-19: Using household data from Bnei Brak, Israel, to estimate the relative susceptibility and infectivity of children. medRxiv. 2020:2020.06.03.20121145. Preprint . https://doi.org/10.1101/ 2020.06.03.20121145

44. James A, Plank MJ, Hendy S, Binny RN, Lustig A, Steyn N. Model-free estimation of COVID-19 transmission dynamics from a complete outbreak. medRxiv. 2020:2020.07.21.20159335. Preprint . https://doi.org/10.1101/2020.07.21.20159335

45. Yung CF, Kam K-Q, Wong MSY, Maiwald M, Tan YK, Tan BH, et al. Environment and personal protective equipment tests for sars-cov-2 in the isolation room of an infant with infection. Ann Intern Med. 2020;173(3):240-2. https://doi.org/10.7326/M200942 PMID: 32236490

46. Xu XK, Liu XF, Wu Y, Ali ST, Du Z, Bosetti P, et al. Reconstruction of transmission pairs for novel Coronavirus Disease 2019 (COVID-19) in mainland China: estimation of superspreading events, serial interval, and hazard of infection. Clin Infect Dis. 2020;71(12):3163-7. https://doi.org/10.1093/ cid/ciaa79o PMID: 32556265

47. Worby CJ, Chaves SS, Wallinga J, Lipsitch M, Finelli L, Goldstein E. On the relative role of different age groups in influenza epidemics. Epidemics. 2015;13:10-6. https://doi.org/10.1016/j. epidem.2015.04.003 PMID: 26097505

48. Ludvigsson JF. Children are unlikely to be the main drivers of the COVID-19 pandemic - A systematic review. Acta Paediatr. 2020;109(8):1525-30. https://doi.org/10.1111/apa.15371 PMID: 32430964

49. Rajmil L. Role of children in the transmission of the COVID-19 pandemic: a rapid scoping review. BMJ Paediatr Open. 2020;4(1):e000722. https://doi.org/10.1136/ bmjpo-2020-000722 PMID: 32596514

50. Park YJ, Choe YJ, Park O, Park SY, Kim YM, Kim J, et al. Contact tracing during coronavirus disease outbreak, South Korea, 2020. Emerg Infect Dis. 2020;26(10):2465-8. https://doi. org/10.3201/eid2610.201315 PMID: 32673193

51. Cui X, Zhang T, Zheng J, Zhang J, Si P, Xu Y, et al. Children with coronavirus disease 2019: A review of demographic, clinical, laboratory, and imaging features in pediatric patients. J Med Virol. 2020;92(9):1501-10. https://doi.org/10.1002/jmv.26023 PMID: 32418216

52. Garazzino S, Montagnani C, Donà D, Meini A, Felici E, Vergine $\mathrm{G}$, et al. Multicentre Italian study of SARS-CoV-2 infection in children and adolescents, preliminary data as at 10 April 2020. Euro Surveill. 2020;25(18):2000600. https://doi. org/10.2807/1560-7917.ES.2020.25.18.2000600 PMID: 32400362

53. Rahimzadeh G, Ekrami Noghabi M, Kadkhodaei Elyaderani F, Navaeifar MR, Enayati AA, Manafi Anari A, et al. COVID-19 infection in Iranian children: a case series of 9 patients. J Pediatr Rev.2020;8(2):139-44. https://doi.org/10.32598/ jpr.8.2.139

54. Shen Q, Guo W, Guo T, Li J, He W, Ni S, et al. Novel coronavirus infection in children outside of Wuhan, China. Pediatr
Pulmonol. 2020;55(6):1424-9. https://doi.org/10.1002/ ppul.24762 PMID: 32259403

55. Zhen-Dong Y, Gao-Jun Z, Run-Ming J, Zhi-Sheng L, ZongQi D, Xiong X, et al. Clinical and transmission dynamics characteristics of 406 children with coronavirus disease 2019 in China: A review. J Infect. 2020;81(2):e11-5. https://doi. org/10.1016/j.jinf.2020.04.030 PMID: 32360500

56. Leclerc QJ, Fuller NM, Knight LE, Funk S, Knight GM. What settings have been linked to SARS-CoV-2 transmission clusters? Wellcome Open Res. 2020;5:83. https://doi. org/10.12688/wellcomeopenres.15889.2 PMID: 32656368

57. Contejean A, Leporrier J, Canouï E, Alby-Laurent F, Lafont E, Beaudeau L, et al. Comparing dynamics and determinants of severe acute respiratory syndrome coronavirus 2 transmissions among healthcare workers of adult and pediatric settings in central Paris. Clin Infect Dis. 2021;72(2):257-64. https://doi. org/10.1093/cid/ciaa977 PMID: 33501952

58. Link-Gelles R, DellaGrotta AL, Molina C, Clyne A, Campagna K, Lanzieri TM, et al. Limited secondary transmission of SARSCoV-2 in child care programs - Rhode Island, June 1-July 31, 2020. MMWR Morb Mortal Wkly Rep. 2020;69(34):1170-2. https://doi.org/10.15585/mmwr.mm6934e2 PMID: 32853185

59. Lopez AS, Hill M, Antezano J, Vilven D, Rutner T, Bogdanow L, et al. Transmission dynamics of COVID-19 outbreaks associated with child care facilities - Salt Lake City, Utah, April-July 2020. MMWR Morb Mortal Wkly Rep. 2020;69(37):1319-23. https:// doi.org/10.15585/mmwr.mm6937e3 PMID: 32941418

6o. The National Collaborating Centre for Methods and Tools (McMaster University). Rapid review update 7: what is the specific role of daycares and schools in COVID-19 transmission? Hamilton: McMaster University; 2020. Available from: https://www.nccmt.ca/knowledge-repositories/ covid-19-rapid-evidence-service

61. Otte Im Kampe E, Lehfeld AS, Buda S, Buchholz U, Haas W. Surveillance of COVID-19 school outbreaks, Germany, March to August 2020. Euro Surveill. 2020;25(38):2001645. https:// doi.org/10.2807/1560-7917.ES.2020.25.38.2001645 PMID: 32975186

62. Ehrhardt J, Ekinci A, Krehl H, Meincke M, Finci I, Klein J, et al. Transmission of SARS-CoV-2 in children aged o to 19 years in childcare facilities and schools after their reopening in May 2020, Baden-Württemberg, Germany. Euro Surveill. 2020;25(36):2001587. https://doi.org/10.2807/1560-7917. ES.2020.25.36.2001587 PMID: 32914746

63. Buonsenso D, De Rose C, Moroni R, Valentini P. SARS-CoV-2 infections in Italian schools: preliminary findings after one month of school opening during the second wave of the pandemic. medRxiv. 2020:2020.10. . https://doi.org/10.1101/2 020.10 .10 .20210328

64. Ismail SA, Saliba V, Lopez Bernal JA, Ramsay ME, Ladhani SN. SARS-CoV-2 infection and transmission in educational settings: cross-sectional analysis of clusters and outbreaks in England. medRxiv. 2020:2020.08.21.20178574. Preprint.

65. Viner RM, Russell SJ, Croker H, Packer J, Ward J, Stansfield $C$, et al. School closure and management practices during coronavirus outbreaks including COVID-19: a rapid systematic review. Lancet Child Adolesc Health. 2020;4(5):397-404. https://doi.org/10.1016/S2352-4642(20)30095-X PMID: 32272089

66. Abdollahi E, Haworth-Brockman M, Keynan Y, Langley JM, Moghadas SM. Simulating the effect of school closure during COVID-19 outbreaks in Ontario, Canada. BMC Med. 2020;18(1):230. https://doi.org/10.1186/s12916-020-01705-8 PMID: 32709232

67. Iwata K, Doi A, Miyakoshi C. Was school closure effective in mitigating coronavirus disease 2019 (COVID-19)? Time series analysis using Bayesian inference. Int J Infect Dis. 2020;99:5761. https://doi.org/10.1016/j.ijid.2020.07.052 PMID: 32745628

68. Viner R, Waddington C, Mytton O, Booy R, Cruz J, Ward J, et al. Transmission of SARS-CoV-2 by children and young people in households and schools: a meta-analysis of population-based and contact-tracing studies. J Infect. 2021.22:S0163-4453(21)00633-2.

69. Armann JP, Unrath M, Kirsten C, Lueck C, Dalpke A, Berner R. Anti-SARS-CoV-2 IgG antibodies in adolescent students and their teachers in Saxony, Germany (SchoolCoviDD19): very low seropraevalence and transmission rates. medRxiv. 2020:2020.07.16.20155143. Preprint.

70. Fontanet A, Grant R, Tondeur L, Madec Y, Grzelak L, Cailleau I, et al. SARS-CoV-2 infection in primary schools in northern France: A retrospective cohort study in an area of high transmission. medRxiv. 2020:2020.06.25.20140178. Preprint https://doi.org/10.1101/2020.06.25.20140178

71. Fontanet A, Tondeur L, Madec Y, Grant R, Besombes C, Jolly N, et al. Cluster of COVID-19 in northern France: A retrospective 
closed cohort study. medRxiv. 2020:2020.04.18.20071134. Preprint.

72. Torres JP, Piñera C, De La Maza V, Lagomarcino AJ, Simian D, Torres B, et al. Severe acute respiratory syndrome coronavirus 2 antibody prevalence in blood in a large school community subject to a coronavirus disease 2019 outbreak: a crosssectional study. Clin Infect Dis. 2021;73(2):e458-65. https:// doi.org/10.1093/cid/ciaa955 PMID: 32649743

73. Brown NE, Bryant-Genevier J, Bandy U, Browning CA, Berns AL, Dott $M$, et al. Antibody responses after classroom exposure to teacher with coronavirus disease, March 2020. Emerg Infect Dis. 2020;26(9):2263-5. https://doi.org/10.3201/ eid2609.201802 PMID: 32597750

74. Desmet S, Ekinci E, Wouters I, Decru B, Beuselinck K, Malhotra-Kumar S, et al. No SARS-CoV-2 carriage observed in children attending daycare centers during the intial weeks of the epidemic in Belgium. J Med Virol. 2021;93(3):1828-31. https://doi.org/10.1002/jmv.26689 PMID: 33230857

75. Indenbaum V, Lustig Y, Mendelson E, Hershkovitz Y, GlatmanFreedman A, Keinan-Boker L, et al. Under-diagnosis of SARSCoV-2 infections among children aged $0-15$ years, a nationwide seroprevalence study, Israel, January 2020 to March 2021. Euro Surveill. 2021;26(48):2101040. https://doi.org/10.2807/15607917.ES.2021.26.48.2101040 PMID: 34857069

76. Cicero A, Potter C, Kirk Sell T, Rivers C, Schoch-Spana M. Filling in the blanks: national research needs to guide decisions about reopening schools in the United States. Baltimore: Johns Hopkins Center for Health Security; 2020. Available from: https://www.centerforhealthsecurity.org/ our-work/pubs_archive/pubs-pdfs/2020/200515-reopeningschools.pdf

77. Langlois EV, Straus SE, Antony J, King VJ, Tricco AC. Using rapid reviews to strengthen health policy and systems and progress towards universal health coverage. BMJ Glob Health. 2019;4(1):eo01178. https://doi.org/10.1136/ bmjgh-2018-001178 PMID: 30899562

78. Henry BM, de Oliveira MHS. Preliminary epidemiological analysis on children and adolescents with novel coronavirus disease 2019 outside Hubei Province, China: an observational study utilizing crowdsourced data. medRxiv. 2020:2020.03.01.20029884. Priprint . https://doi.org/10.1101/ 2020.03.01.20029884

79. Liao J, Fan S, Chen J, Wu J, Xu S, Guo Y, et al. Epidemiological and clinical characteristics of COVID-19 in adolescents and young adults. medRxiv. 2020:2020.03. . https://doi org/10.1101/2020.03.10.20032136

\section{License, supplementary material and copyright}

This is an open-access article distributed under the terms of the Creative Commons Attribution (CC BY 4.0) Licence. You may share and adapt the material, but must give appropriate credit to the source, provide a link to the licence and indicate if changes were made.

Any supplementary material referenced in the article can be found in the online version.

This article is copyright of the authors or their affiliated institutions, 2022. 